\title{
RESEARCH ON A WIND LIDAR WITHOUT BLIND ZONE BASED ON THE TECHNOLOGIES OF PSEUDO RANDOM CODE PHASE MODULATION AND HETERODYNE DETECTION
}

\author{
FU YANG ${ }^{1 *}$, ZHENG LIU ${ }^{1}$, XIAOPENG ZHU ${ }^{2}$ \\ 1: College of Science, Donghua University,, Shanghai 201620, China \\ 2: Shanghai Institute of Optics and Fine Mechanics, Chinese Academy of Science, 201800, China \\ *Email: fuyang@dhu.edu.cn
}

\begin{abstract}
In this measurement method, a wind lidar system with no blind zone based on the technologies of heterodyne detection and pseudorandom code phase modulation is proposed. The simulation results show the feasibility of the method. $15 \mathrm{~m}$ range resolution and $7.75 \mathrm{~cm} / \mathrm{s}$ line of sight (LOS) wind velocity resolution can be achieved from $0 \mathrm{~m}$ to $300 \mathrm{~m}$, when the laser transmitted power is $2 \mathrm{~mW}$, the transmitted pulse length is $100 \mathrm{us}$, the receiving telescope aperture is $2 \mathrm{~cm}$, and an accumulation times of 10 . The simulation results also show the positive effect of the transmitted pulse length.
\end{abstract}

Key words: wind lidar, heterodyne detection, blind zone

\section{INTRODUCTION}

An accurate measurement of the wind velocity plays a key role in the fields of weather forecast, aviation safety, air pollution control, wind energy utilization and so on[1]. The direct wind detection system extracts the wind LOS velocity through an optical frequency discriminator [2] while the coherent wind detection system get the wind LOS velocity through Doppler frequency [3]. No matter which detection mechanism, the system exists an area of blind zone due to the end reflection signal. In addition, in order to obtain better range resolution, the pulse width of the laser should be short, so a huge peak power is required. In this paper, a wind lidar measurement system with no blind zone is proposed. The high range resolution is realized by random phase modulation, the high velocity resolution is realized by transmitted long pulse length, and high sensitivity is realized by adopting heterodyne detection.

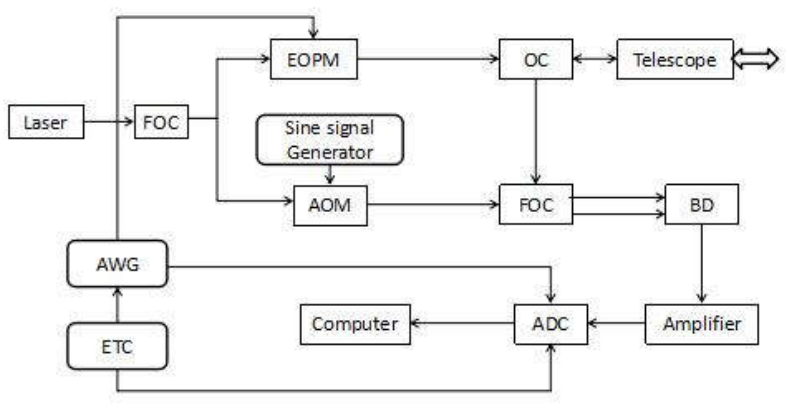

Figure 1 A schematic view of the system

\section{METHODOLOGY}

\subsection{The lidar system}

The schematic of the system is shown in Fig.1. [4]

FOC: fiber optic coupler;

EOPM: electro-optic phase modulator;

AOM: acousto-optic modulator;

OC: optical circulator;

BD: Balance detector;

ADC: dual channel AD capture card;

AWG: Arbitrary waveform generator;

ETC: external trigger circuit

The system uses an eye-safe $1550 \mathrm{~nm}$ narrow linewidth fiber laser as the light source. The output laser is split into two beams by a $1 \times 2$ FOC. One part serves as reference light and the other as signal light, and the other serves as local oscillator (LO). The EOPM produces phase modulation of the signal light according to the PRC sequence, and its drive signal can be provided by the AWG. The output signal after PRC phase modulation is shown in Eq. 1

$$
\mathrm{S}=A_{T} \cos \left[\omega_{c}+P_{R C}^{\prime}(t) \pi+\emptyset_{1}\right]
$$

Where $A_{T}$ is the amplitude of the laser, $\omega_{c}$ is the carrier frequency, $\varnothing_{1}$ is an unchanged random 
phase, and the PRC sequence $P_{R C}^{\prime}(t)$ is shown in Eq. 2:

$$
P_{R C}^{\prime}(t)=\sum_{n=-\infty}^{n=+\infty} b_{n} p\left(t-n T_{0}\right)
$$

Where $b_{n}$ is a pseudo-random code, and the value is 0 or 1 with the same probability. The system uses the M-sequence PRC, $p(t)$ is the gate function shown in Eq. 3:

$$
\mathrm{p}(\mathrm{t})= \begin{cases}1, & |\mathrm{t}| \leq T_{0} / 2 \\ 0, & |\mathrm{t}|>T_{0} / 2\end{cases}
$$

Where $T_{0}=1 / \mathrm{F}$, is the PRC code width. It is the inverse of modulation rate. The local oscillator (LO) signal is

$$
\mathrm{L}=A_{L O} \cos \left[\left(\omega_{c}+\omega_{m}\right) t+\emptyset_{2}\right]
$$

Where $A_{L O}$ is the amplitude of the LO, $\omega_{m}$ is the frequency shift of the AOM, and $\emptyset_{2}$ is an unchanged random phase. The drive signal of the $\mathrm{AOM}$ is provided by a signal generator. The modulated signal light is transmitted by a telescope through port 1 of the OC. The return signal light is received by the same telescope, and coherent with the LO through port 3 of OC in a FOC. The return signal carries the LOS wind velocity. The coherent signal is detected by a BD. After amplification, the signal is sampled by channel 1 of the dual-channel ADC. The other channel of the ADC samples the output signal of the AWG which is used to offer variable delay pseudo random signals needed in the signal process. The ADC and the AWG generator are triggered by the same external trigger circuit (ETC) to ensure time synchronization.

The coherent signal is a phase-discontinuous triangular wave signal, whose frequency is decided by the AOM and the Doppler frequency shift.

The output of $\mathrm{BD}$ can be expressed as:

$$
\begin{aligned}
i_{S}(\mathrm{t})= & A_{S} A_{L O} \cos \left[\left(\omega_{m}+\omega_{d}\right)\left(t-\frac{2 R}{c}\right)+\right. \\
& \left.P_{R C}^{\prime}\left(t-\frac{2 R}{c}\right) \pi+\varphi_{3}\right]
\end{aligned}
$$

Where $\mathrm{R}$ is the distance between the detected wind and the system, $\mathrm{c}$ is the speed of light, and $\varphi_{3}$ is an unchanged random phase. $A_{S}$ is the amplitude of the echo signal, and its relationship with the echo signal power $P_{\text {sig }}$ is $A_{S}=\sqrt{P_{\text {sig }}}$. $P_{\text {sig }}=\eta_{\text {atm }} \eta_{\text {sys }} P_{T} c T_{0} \beta \frac{\pi D^{2}}{4 R^{2}}, \eta_{\text {atm }}$ and $\eta_{\text {sys }}$ are the optical transmittance of the atmosphere and radar system, respectively, $P_{T}$ is the transmitted laser peak power, $c$ is the speed of light, $T_{0}$ is the length of each PRC code, $\beta$ is backscatter coefficient. $\mathrm{D}$ is the telescope diameter, and $\mathrm{R}$ is the range of the wind. $\varphi_{3}$ is an unchanged random phase.

According to the character of cosine function, the Eq. 5 can be expressed as Eq. 6 :

$$
\begin{gathered}
i_{s}(\mathrm{t})=A_{S} A_{L O} P_{R C}\left(t-\frac{2 R}{c}\right) \cos \left[\left(\omega_{m}+\right.\right. \\
\left.\left.\omega_{d}\right) t+\varphi_{4}\right]
\end{gathered}
$$

Where $\varphi_{4}$ is an unchanged random phase.

The acquired signals from the 2 channels of the ADC are simultaneously multiplied and FFT transformed, the final signal is shown in Eq. (7)

$$
\begin{gathered}
S_{I G}=\mathcal{F}(\mathrm{Y})=\mathcal{F}\left[i_{s}(\mathrm{t}) \times P_{R C}\left(\mathrm{t}-\mathrm{n} T_{0}\right)\right] \\
=A_{S} A_{L O} \mathcal{F}\left[P_{R C}(\mathrm{t}-2 \mathrm{R} / \mathrm{c}) \times P_{R C}\left(\mathrm{t}-\mathrm{n} T_{0}\right)\right. \\
\left.\times \cos \left(\left(\omega_{m}+\omega_{d}\right) t+\emptyset_{4}\right)\right] \\
\mathrm{n}=1,2,3 \ldots \ldots \mathrm{N}
\end{gathered}
$$

For each time delay amount $\mathrm{nT}_{0}$, the wind information located at around $\mathrm{cnT}_{0} / 2$ can be extracted. So the range resolution is $\mathrm{cT}_{0} / 2$, which is $15 \mathrm{~m}$ in our simulation. The corresponding spectral peak $f$ can be obtained. The difference of $f$ with respect to $\omega_{m}$ is the Doppler frequency shift caused by the wind LOS velocity. The relation between the Doppler frequency shift and LOS wind velocity $v$ is $v=\frac{\lambda \omega_{d}}{2}$. If $v>0$, it means the wind moves towards the system. Otherwise, it means the opposite direction. All of the system signal processing can be done in the computer.

For the end face reflection signal, we know the distance of it, and the Doppler frequency is 0 . So we remove the peak, which is contributed by the end face reflection, in the FFT spectrum when the delay range $\mathrm{cnT}_{0} / 2$ matches the end face.

\section{RESULTS}


The system is simulated by using Matlab. The simulation parameters are shown in Tab. 1 .

Table 1

\begin{tabular}{|c|c|}
\multicolumn{1}{c}{ Parameter } & Value \\
\hline$P_{T}$ & $0.2 \mathrm{~mW}$ \\
\hline$P_{L O}$ & $0.1 \mathrm{~m}$ \\
\hline $\begin{array}{c}\text { D (Telescope } \\
\text { diameter) }\end{array}$ & $10^{-6}$ \\
\hline $\begin{array}{c}\text { Br (End reflection } \\
\text { coefficient) }\end{array}$ & $10 \mathrm{MHz}$ \\
\hline $\begin{array}{c}\text { Backscatter coefficient } \\
\text { F ( Phase Modulation } \\
\text { frequency) }\end{array}$ & $3.5 \times 10^{-6} \mathrm{~m}^{-1} \cdot S_{r}^{-1}$ \\
\hline $\begin{array}{c}\text { Line of sight (LOS) } \\
\text { wind velocity } \\
\text { AOM }\end{array}$ & $50 \mathrm{MHz}$ \\
\hline $\begin{array}{c}\text { T (The transmitted } \\
\text { pulse length) }\end{array}$ & $50 \mathrm{us}$ \\
\hline & \\
\hline
\end{tabular}

The relation between the intensity of the ADC channel 1 (which is in proportional to the output of $\mathrm{BD}$ ) signal and the range is shown in Fig. 2

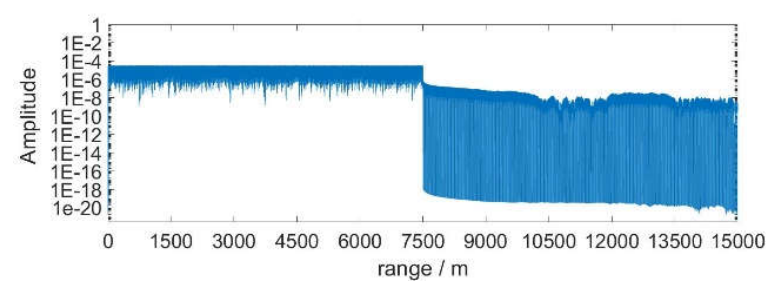

Figure 2 The amplitude of the ADC channel 1 versus the wind range

We use logarithmic coordinate for the $\mathrm{Y}$-axis. It can be seen that the intensity of the first half section in Fig. 2 is much stronger than the second half section. Because we use the long transmitting pulse length which is 500 us in the simulation, the end face reflection effect lasts 50 us (corresponding to $7500 \mathrm{~m}$ for the range in air). The intensity of the first half is mostly contributed by the end face reflection. In the second half section, we can see the attenuation of the return wind signal due to the increase of range.

After an accumulation times of 25 , the final signal for the range of $1 \mathrm{~m}$ and $150 \mathrm{~m}$ are shown in FIG.3

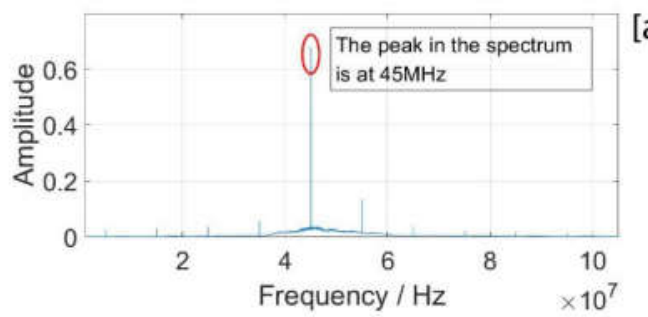

[a]

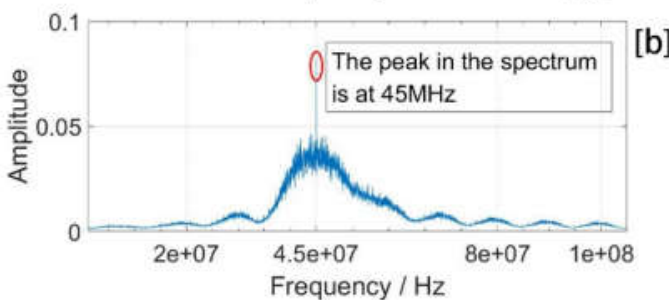

Figure 3 The final signal spectrum for the range of (a) $1 \mathrm{~m}$ (b) $150 \mathrm{~m}$

From Fig. 3, it is observed that the wind information at $1 \mathrm{~m}$ and $150 \mathrm{~m}$ can both be detected clearly. Fig. 3(a) shows clearly that the end face signal effect can be removed. The peak frequency deviates from the $50 \mathrm{MHz}(\mathrm{AOM}$ frequency shift) is due to doppler effect. The LOS wind velocity is $3.875 \mathrm{~m} / \mathrm{s}$.

The LOS wind velocity resolution is decided by the transmitted pulse length.

$$
d v=\frac{\lambda}{4 \mathrm{~T}}
$$

Where $d v$ is The LOS wind velocity resolution, $T$ is the transmitted pulse length. According to the simulation parameters in Tab.1, the LOS wind velocity resolution is $0.775 \mathrm{~cm} / \mathrm{s}$.

The frequency shift of the AOM determines the wind detection range according to $V=$ $\frac{\lambda \cdot A O M}{2}$. Based on Tab. 1, the LOS wind velocity range is $0 \sim 38.75 \mathrm{~m} / \mathrm{s}$, which is adequate for wind detection.

The relationship between the maximum detection range and the accumulation times under 
variable sequence length is shown in Fig.4 In the simulation, the effects of noise have been considered.

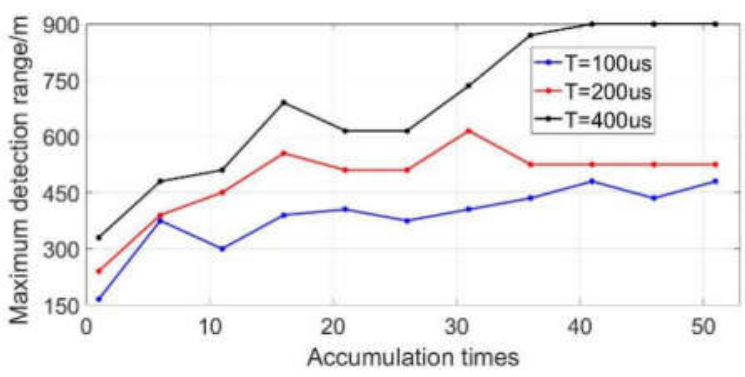

Figure 4 The relationship between the maximum detection range and the accumulation times under variable sequence length

From Fig. 4, it's observed that the transmitted pulse length plays a positive effect on the maximum detection range. And the effect of accumulative times will tend to saturation.

The relationship between the maximum detection range and the transmitted laser peak power under variable sequence length is shown in Fig.5. And the accumulative times is set at 50 .

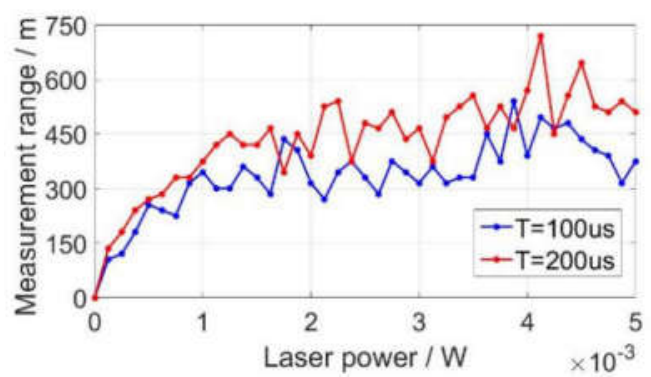

Figure 5 The relationship between the maximum detection range and the transmitted laser peak power under variable sequence length

From Fig. 5, it is observed that the transmitted pulse length plays a positive effect on the maximum detection range. And the effect of laser transmitted peak power will tend to saturation.

At the same time, this system does not require too much laser power. $2 \sim 3 \mathrm{~mW}$ transmitted laser peak power can detect the wind peak power of several hundred meters.
A wind lidar without blind zone based on the technologies of pseudo random code phase modulation and heterodyne detection is demonstrated in the simulation. The high range resolution is realized by fast modulation rate, the high velocity resolution is realized by transmitting long pulse length, and high sensitivity is realized by adopting heterodyne detection. $0.775 \mathrm{~cm} / \mathrm{s}$ line of sight (LOS) wind velocity resolution and range resolution can be achieved from $0 \mathrm{~m}$ to 300 $\mathrm{m}$, when the laser transmitted power is $2 \mathrm{~mW}$, the transmitted pulse length is 100 us, the receiving telescope aperture is $2 \mathrm{~cm}$, and an accumulative times of 10. Unlike conventional systems, the system has neither close-range blind spots nor excessive peak power requirements.

\section{ACKNOWLEDGE}

The research is sponsored by the opening foundation of Chinese Academy of Science Key Laboratory of Space Laser Information Transportation and Detection Technology.

\section{REFERENCES}

[1] Zhou Yanzong, Wang Chong, Liu Yanping, Xia Haiyun. Research Progress and Application of Coherent Wind Lidar[J]. Progress in Laser and Optoelectronics, 2019, (02): 9-26.

[2] Sun Dongsong, Yang Zhao, Xue Guogang. Performance Analysis of Direct Detection of Wind Lidar[J].Infrared and Laser Engineering,2003(02):115-117+126.

[3] Pan Jingyan, Yan Shuangyang, Liu Guo, Dong Guangyan, Zhang Pengfei, Chen Jing. Wind field measurement technology of coherent laser wind radar[J].Infrared and Laser Engineering,2013,42(07):1720-1724.

[4] Fu Yang, Yan He, Weibiao Chen, and Yage Zhan IEEE PHOTONICS TECHNOLOGY LETTERS, VOL. 26, NO. 23, DECEMBER 1, 2014

\section{Conclusion}

